[0212-7199 (2007) 24: 4; pp 168-172] ANALES DE MEDICINA INTERNA Copyright (C) 2007 ARAN EDICIONES, S.L.

AN. MED. INTERNA (Madrid) Vol. 24, N. $^{\circ} 4$, pp. $168-172,2007$

\title{
Impacto del sexo en el síndrome de lipodistrofia en pacientes con infección por el VIH y su asociación con factores de riesgo cardiovascular
}

\author{
M. L. SORLI REDÓ, H. KNOBEL FREUD, M. MONTERO, C. JERICÓ ALBA, \\ A. GUELAR GRIMBERG, J. PEDRO-BOTET MONTOYA
}

Servicio de Medicina Interna y Enfermedades Infecciosas. Hospital del Mar. Universidad Autónoma de Barcelona. Barcelona

SEX INFLUENCE IN LIPODYSTROPHY OF HIV-INFECTED PATIENTS AND ITS ASSOCIATION WITH CARDIOVASCULAR RISK FACTORS

\begin{abstract}
RESUMEN
Objetivo: Evaluar el impacto del sexo en la presencia del síndrome de lipodistrofia en una población de pacientes infectados por el VIH y su asociación con los factores de riesgo cardiovascular.

Pacientes y método: Estudio transversal que incluyó a los pacientes de 20 años de edad o mayores, con infección por el VIH atendidos en la consulta externa del Servicio de Medicina Interna y Enfermedades Infecciosas del Hospital del Mar de Barcelona durante el año 2.003. Se evaluaron las características clínico-epidemiológicas de la infección por el $\mathrm{VIH}$, la presencia de lipodistrofía y los factores de riesgo cardiovascular.

Resultados: De los 710 pacientes incluidos en el estudio, las mujeres representaban el $28 \%$ de la serie. Los varones con lipodistrofia presentaron una mayor prevalencia de hipertensión arterial, hipercolesterolemia, hipoalfalipoproteinemia e hipertrigliceridemia comparado con los que no presentaban lipodistrofia $(22,8$ vs. $11,2 \%, \mathrm{p}=0,000 ; 20,6$ vs. $9,3 \%, \mathrm{p}=$ 0,$001 ; 39,7$ vs. $30 \%, \mathrm{p}=0,03$ y 56,6 vs. $40,9 \%, \mathrm{p}=0,0001$, respectivamente). En las mujeres, la presencia de lipodistrofia se acompañó de una mayor prevalencia de obesidad central e hipertrigliceridemia con respecto a la ausencia de lipodistrofia. En los varones predominó el patrón de lipoatrofia $(24,9$ vs. $12,6 \%, \mathrm{p}=0,0001)$, y en las mujeres el lipoacúmulo $(12,3$ vs. $22,6 \%, \mathrm{p}=0,0001)$. Además, las mujeres eran más jóvenes, tenían una mayor prevalencia de tabaquismo, antecedentes familiares de cardiopatía isquémica prematura y de obesidad central, y una menor prevalencia de hipertensión arterial y de hipoalfalipoproteinemia que los varones con lipodistrofia $(42,1 \pm 8$ años vs. 44,8 $\pm 9,9$ años, $\mathrm{p}=0,03$; 77,5 vs. $64 \%, \mathrm{p}=0,04 ; 22,5$ vs. $9 \%, \mathrm{p}=0,003 ; 31$ vs. $8,5 \%, \mathrm{p}=0,0001$; 9,9 vs. $22,8 \%, \mathrm{p}=0,01 ; 25,4$ vs. $39,7 \%, \mathrm{p}=0,03$, respectivamente).

Conclusiones: El presente estudio demuestra que el impacto del sexo en la lipodistrofia de los pacientes con infección por el VIH no sólo afecta al patrón de lipodistrofia, sino también al perfil de riesgo cardiovascular.
\end{abstract}

PALABRAS CLAVE: Tratamiento antirretroviral. VIH. Lipodistrofia. Factores de riesgo cardiovascular

\section{ABSTRACT}

Objective: To evaluate the influence of sex in human immunodeficiency virus (HIV)-infected patients with lipodystrophy and its association with cardiovascular risk factors.

Patients and method: A cross-sectional study was conducted in HIVinfected patients aged 20 or over managed at the outpatient Infectious Disease Unit in 2003. Clinical and epidemiological characteristics of HIV infection, lipodystrophy and cardiovascular risk factors were evaluated.

Results: Of the 760 patients included in the study, women comprised 28\%. Men with lipodystrophy had a higher prevalence of hypertension, hypercholesterolemia, hypoalphalipoproteinemia and hypertriglyceridemia than those without lipodystrophy. Women with lipodystrophy had a higher prevalence of central obesity and hypertriglyceridemia compared with those without lipodystrophy (22.8 vs. 11.2\%, $p=0.000 ; 20.6$ vs. $9.3 \%, p=0.001 ; 39.7$ vs. $30 \%, p=0.03$ y 56.6 vs. $40.9 \%, p=0.0001$, respectively). The lipoatrophy pattern was predominant in men (24.9 vs. $12.6 \%, p=0.0001)$ and lipoaccumulation forms in women (12.3 vs. $22.6 \%, p=0.0001)$. Furthermore, women were younger, had a higher prevalence of smoking, family history of premature coronary heart disease and central obesity, and a lower prevalence of hypertension and hypoalphalipoproteinemia than men with lipodystrophy $(42.1 \pm 8$ years vs. $44.8 \pm 9.9$ years, $p=0.03 ; 77.5$ vs. $64 \%, p=0.04 ; 22.5$ vs. $9 \%, p=$ $0.003 ; 31$ vs. $8.5 \%, p=0.0001 ; 9.9$ vs. $22.8 \%, p=0.01 ; 25.4$ vs. $39.7 \%$, $p=0.03$ ).

Conclusions: This study demonstrated that the influence of sex in lipodystrophy in HIV-infected patients affects not only the lipodystrophy pattern, but also the cardiovascular risk profile.

KEY WORDS: Antiretroviral therapy. HIV. Lipodystrophy. Cardiovascular risk factors.

Sorli Redó ML, Knobel Freud H, Montero M, Jericó Alba C, Guelar Grimberg A, Pedro-Botet Montoya J. Impacto del sexo en el síndrome de lipodistrofia en pacientes con infección por el VIH y su asociación con factores de riego cardiovascular. An Med Interna (Madrid) 2007; 24: $168-172$.

\section{INTRODUCCIÓN}

Las estadísticas vitales demuestran que la mortalidad coronaria aumenta de forma constante y continuada con la edad en ambos sexos (1). Aunque los mecanismos fisiopatológicos, los factores de riesgo, la presentación clínica y el pronóstico de la enfermedad cardiovascular son diferentes según el sexo (2-4), las causas subyacentes son complejas y no bien

Trabajo aceptado: 12 de diciembre de 2006 
aclaradas. En los últimos años se ha profundizado en la relación existente entre la obesidad visceral o abdominal y la enfermedad cardiovascular tanto en el varón como en la mujer $(5,6)$, sugiriendo que las disimilitudes en la distribución de la grasa corporal podrían explicar parte de las diferencias del riesgo cardiovascular según el sexo (7).

Por otra parte, en los pacientes con infección por el virus de la inmunodeficiencia humana (VIH), el tratamiento antirretroviral de gran actividad (TARGA) ha modificado favorablemente la evolución natural de la enfermedad con una drástica disminución de la morbi-mortalidad asociada a las enfermedades oportunistas $(8,9)$. Sin embargo, su uso se ha relacionado con alteraciones del metabolismo lipoproteico y de los hidratos de carbono, y anomalías en la distribución de la grasa corporal, todas ellas agrupadas bajo la denominación de síndrome de lipodistrofia (10). Estas complicaciones atribuidas a la toxicidad de los fármacos antirretrovirales, podrían explicar al menos en parte el aumento del riesgo cardiovascular en estos pacientes $(11,12)$. Aunque el papel de los distintos tipos de fármacos y de cada fármaco en particular no está completamente esclarecido, se ha propuesto un efecto aditivo en el desarrollo de la lipodistrofia; los inhibidores de la proteasa en forma de lipoacúmulo y los inhibidores de nucleósidos en forma de lipoatrofia $(13,14)$. Además, las mujeres con infección por el VIH presentan un mayor riesgo de desarrollar alteraciones en la distribución de la grasa corporal relacionadas con la terapia antirretroviral (15). A pesar de que las mujeres representan la mitad de la población infectada por el VIH, y de que el sexo sigue siendo un factor independiente de riesgo cardiovascular en esta población (16), cabe destacar su escasa representatividad en la mayoría de estudios clínico-epidemiológi$\cos 12,17)$. Por dicho motivo, en el presente estudio se evaluó el impacto del sexo en la presencia del síndrome de lipodistrofia en una población de pacientes infectados por el VIH y su asociación con los principales factores de riesgo cardiovascular.

\section{PACIENTES Y MÉTODOS}

Pacientes. Estudio transversal de los pacientes con infección por el VIH atendidos en la consulta externa del Servicio de Medicina Interna y Enfermedades Infecciosas del Hospital del Mar de Barcelona entre enero y diciembre de 2003. Se incluyeron a los pacientes de 20 años de edad o mayores, que no presentaran signos de infección activa o enfermedad definitoria de SIDA en los 3 meses previos a su inclusión, ni se hubiese interrumpido el TARGA por su posible impacto en los parámetros antropométricos y bioquímicos. El protocolo de estudio, aprobado por el Comité de Ética del hospital, incluía un examen físico y una extracción sanguínea para estudio de parámetros de laboratorio.

De cada paciente se evaluaron los factores de riesgo cardiovascular según las recomendaciones del Panel III del National Cholesterol Education Program (NCEP) (18). Se consideraron la edad, el sexo, los antecedentes familiares de cardiopatía isquémica prematura, el consumo de cigarrillos, y si habían presentado manifestaciones clínicas de enfermedad cardiovascular. Se efectuó el diagnóstico de hipertensión arterial si la presión arterial era $\geq 140 / 90$ mm Hg o recibía fármacos antihipertensivos (19). Asimismo, se realizó el diagnóstico de diabetes mellitus cuando la glucemia en ayunas era $\geq$ $126 \mathrm{mg} / \mathrm{dl}$ o si recibía fármacos hipoglucemiantes (20). Se evaluó la concentración sérica de colesterol total y del contenido en las fracciones lipoproteicas.

Con respecto a la infección por el VIH se valoraron el grupo de riesgo para la transmisión de la enfermedad clasificado como usuario de drogas por vía parenteral, sexual y desconocido u otros, el tiempo transcurrido desde el diagnóstico de la infección, el recuento de linfocitos CD4 nadir, haber presentado enfermedad definitoria de SIDA (21) y la exposición a tratamiento antirretroviral clasificada como naïve o en tratamiento. En los pacientes que habían recibido el TARGA se consideró además el tiempo acumulado de tratamiento y si éste incluía inhibidores de la proteasa.

El examen físico incluyó la medida del peso, la talla, y el perímetro de cintura abdominal, así como la determinación de la presión arterial según las técnicas estandarizadas. La presencia de lipodistrofia se evaluó mediante la percepción por parte del paciente de cambios en la distribución de la grasa corporal y la valoración por un único investigador (H.K.), desconocedor de la información clínica, que la clasificó en lipoatrofia cuando existía una disminución de la grasa subcutánea periférica (cara, extremidades superiores o inferiores y nalgas), lipohipertrofia si había acúmulo de grasa a nivel troncular (peri o intraabdominal, dorsocervical y mamaria) y en formas mixtas. La presencia de obesidad central se definió como un perímetro de cintura abdominal superior a $102 \mathrm{~cm}$ en los varones y a $88 \mathrm{~cm}$ en las mujeres (18).

Determinaciones. A todos los pacientes incluidos en el estudio se les realizó una extracción de sangre venosa tras ayuno de 12 horas. Se determinaron las concentraciones de colesterol total y triglicéridos mediante métodos enzimáticos en un analizador automático Cobas Mira (Baxter Diagnostics AG, Düdingen, Suiza) y la del colesterol de las lipoproteínas de alta densidad (cHDL) mediante separación por precipitación con ácido fosfotúngstico y cloruro de magnesio. El colesterol de las lipoproteínas de baja densidad (cLDL) se calculó utilizando la fórmula de Friedewald excepto en los pacientes con trigliceridemia superior a $400 \mathrm{mg} / \mathrm{dl}$ en los que se realizó ultracentrifugación de lipoproteínas. La glucemia se determinó por el método de la glucosa oxidasa. El recuento de linfocitos CD4 y la carga viral (Nuclisens Easy Q HIV-1, Biomerieux, Boxtel, Holanda) se realizaron en el momento del estudio; el recuento de linfocitos CD4 nadir y la carga viral basal se recogieron de la historia clínica de cada paciente.

Análisis estadístico. Las variables cuantitativas se presentan como media y desviación estándar o mediana y rango intercuartil según su distribución, y las cualitativas como estimaciones porcentuales. Se utilizó la prueba de la t de Student para la comparación de medias, la U de Mann-Whitney para la comparación no paramétrica y el test de la Ji al cuadrado para el análisis de las variables categóricas, tomando como significativas las diferencias con un valor de la $\mathrm{p}<0,05$. Para el análisis de los resultados se empleó el programa estadístico SPSS, versión 12.0 para Windows.

\section{RESULTADOS}

De los 1.016 pacientes controlados durante el 2003 en la consulta externa del Servicio de Medicina Interna y Enfermedades Infecciosas, 209 se excluyeron por el criterio de la edad, haber presentado signos de infección activa, precisar ingreso hospitalario, o por interrupción de la tera- 
pia antirretroviral. De los 807 pacientes elegibles, sólo 710 (88\%), completaron el protocolo de estudio. En la tabla I se exponen las características demográficas, antropométricas y de la infección por el VIH, así como la glucemia en ayunas y el perfil lipoproteico de los 710 pacientes incluidos en el estudio. Las mujeres representaban el $28 \%$ de la serie, eran significativamente más jóvenes y presentaban un índice de masa corporal, perímetro de cintura abdominal, cifras de presión arterial sistólica, glucemia basal en ayunas y trigliceridemia menores que los varones. Las características inmunológicas y virológicas fueron muy similares en ambos sexos, excepto en la vía de adquisición de la infección.

En la tabla II se describe la prevalencia de alteraciones en la distribución de la grasa corporal según el sexo, y su relación con los principales factores de riesgo cardiovascular. Los varones con lipodistrofia mostraron una mayor prevalencia de hipertensión arterial, hipercolesterolemia, hipoalfalipoproteinemia e hipertrigliceridemia comparado con los que no presentaban lipodistrofia. En las mujeres, la presencia de lipodistrofia se acompañó de una mayor prevalencia de obesidad central e hipertrigliceridemia, con una tendencia a presentar bajas concentraciones de cHDL con respecto a la ausencia de lipodistrofia. Asimismo, tanto los varones como las mujeres con lipodistrofia tenían una edad superior a sus respectivos homólogos sin lipodistrofia.

$\mathrm{Al}$ analizar las características diferenciales entre los varones y las mujeres ambos con lipodistrofia, se observó que en los primeros predominaba el patrón de lipoatrofia $(24,9$ vs. $12,6 \%, p=0,0001)$, mientras que en las mujeres fue más prevalente el lipoacúmulo (patrón de lipohipertrofia y formas mixtas) $(12,3 v s .22,6 \%, \mathrm{p}=0,0001)$. Además, las mujeres eran más jóvenes, tenían una mayor prevalen- cia de tabaquismo, antecedentes familiares de cardiopatía isquémica prematura y de obesidad central, y una menor prevalencia de hipertensión arterial y de hipoalfalipoproteinemia que los varones con lipodistrofia (Tabla II).

\section{DISCUSIÓN}

La infección por el VIH en la mujer es un problema creciente en el mundo. Hasta no hace mucho, el VIH se consideraba una infección casi exclusiva de los varones por lo que la mayoría de los estudios clínicos se centraba en este grupo de población. Sin embargo, en el momento actual, existe un progresivo interés en conocer si la infección por el virus y sus consecuencias presentan rasgos diferenciales según el sexo (22-24). En la población estudiada, los varones y las mujeres mostraron características muy similares en lo referente a la infección por VIH, y superponibles a las descritas en otras series europeas y norteamericanas $(11,16,17)$.

Al analizar la prevalencia de los factores de riesgo cardiovascular según el sexo, las mujeres mostraron una menor prevalencia de hipertensión arterial y un perfil lipoproteico menos aterogénico que el de los varones. Es muy probable que la menor edad de las mujeres de esta población ejerza una influencia decisiva, ya que no debemos olvidar que la mujer premenopáusica está relativamente protegida de la enfermedad cardiovascular, en parte por el efecto de los estrógenos en el metabolismo lipoproteico. De hecho, los resultados del estudio DAD (16) indican que el sexo femenino es un factor protector para el riesgo de infarto de miocardio.

La prevalencia global de lipodistrofia en cualquiera de sus formas clínicas, no difirió de la descrita en estudios previos

\section{TABLA I}

CARACTERÍSTICAS DEMOGRÁFICAS, GLUCEMIA, PERFIL LIPOPROTEICO Y DE LA INFECCIÓN POR VIH DE LOS 710 PACIENTES SEGÚN EL SEXO

\begin{tabular}{lccc}
\hline & $\begin{array}{c}\text { Varones } \\
(n=511)\end{array}$ & $\begin{array}{c}\text { Mujeres } \\
(n=199)\end{array}$ & $P$ \\
\hline Edad, años & $43,0 \pm 9,4$ & $39,5 \pm 8,2$ & 0,0001 \\
Perímetro cintura, (cm) & $86,7 \pm 9,9$ & $80,9 \pm 11,4$ & 0,0001 \\
IMC, kg/m & $23,7 \pm 3,9$ & $22,7 \pm 3,7$ & 0,003 \\
PAS, mm Hg & $119,5 \pm 14,1$ & $112,7 \pm 13,6$ & 0,0001 \\
Glucemia, mg/dl & $98,5 \pm 22$ & $94,0 \pm 17,2$ & 0,009 \\
Colesterol total, mg/dl & $189,9 \pm 48,1$ & $191,7 \pm 48,3$ & 0,6 \\
Colesterol LDL, mg/dl & $113 \pm 37,8$ & $113,0 \pm 39,8$ & 0,9 \\
Colesterol HDL, mg/dl & $46,0 \pm 12,2$ & $53,4 \pm 15$ & 0,0001 \\
Triglicéridos, mg/dl & $184,7 \pm 143,7$ & $153,4 \pm 114,1$ & 0,006 \\
Ex UDVP & $199(39 \%)$ & $94(47 \%)$ & 0,05 \\
Estadio A & $292(57,1 \%)$ & $199(56,3 \%)$ & 0,8 \\
Mediana CD4 nadir, cels/ml & $201(81-334)$ & $200(62-323)$ & 0,1 \\
Mediana incremento CD4 & $248(108-439)$ & $214(83-420)$ & 0,1 \\
Viremia < 50 cp/ml & $316(61,8 \%)$ & $126(63,6 \%)$ & 0,6 \\
No expuestos a TARGA & $61(12 \%)$ & $23(11,6 \%)$ & 0,9 \\
Expuestos a IP & $336(65,9 \%)$ & $131(65,8 \%)$ & 0,9 \\
Tiempo exposición a TARGA, meses & $78(42,5-104)$ & $81(40,5-108)$ & 0,9 \\
\hline
\end{tabular}

IMC: índice de masa corporal; PAS: presión arterial sistólica; LDL: lipoproteínas de baja densidad; HDL: lipoproteínas de alta densidad; UDVP: usuarios drogas por vía parenteral; TARGA: tratamiento antirretroviral de gran actividad; IP: inhibidor de proteasas. 
TABLA II

COMPARACIÓN DE FACTORES DE RIESGO CARDIOVASCULAR EN FUNCIÓN DEL PATRÓN DE DISTRIBUCIÓN DE LA GRASA CORPORAL SEGÚN EL SEXO

\begin{tabular}{lcccccc}
\hline & \multicolumn{2}{c}{$\begin{array}{c}\text { Varones } \\
(\mathrm{n}=511)\end{array}$} & $\mathrm{P}$ & \multicolumn{2}{c}{$\begin{array}{c}\text { Mujeres } \\
(\mathrm{n}=199)\end{array}$} \\
\hline & Con lipodistrofia & Sin lipodistrofia & & Con lipodistrofia & Sin lipodistrofia \\
\hline Edad* & $44,8 \pm 9,3$ & $41,8 \pm 9,3$ & 0,001 & $42,1 \pm 8,0^{\mathrm{a}}$ & $38,0 \pm 7,9$ & 0,001 \\
Tabaco & $121(64 \%)$ & $214(66,5 \%)$ & 0,6 & $55(77,5 \%)^{\mathrm{b}}$ & $89(69,5 \%)$ & 0,2 \\
AF Cl $^{*}$ & $17(9 \%)$ & $44(13,7 \%)$ & 0,1 & $16(22,5 \%)^{\mathrm{c}}$ & $22(17,2 \%)$ & 0,2 \\
ECV & $8(4,2 \%)$ & $14(4,3 \%)$ & 0,9 & $1(1,4 \%)$ & $0(0 \%)$ & 0,3 \\
Obesidad central & $16(8,5 \%)$ & $27(8,4 \%)$ & 0,9 & $22(31 \%) \mathrm{d}$ & $24(18,8 \%)$ & 0,05 \\
HTA & $43(22,8 \%)$ & $36(11,2 \%)$ & 0,000 & $7(9,9 \%)$ & $7(5,5 \%)$ & 0,2 \\
Diabetes & $13(6,9 \%)$ & $11(3,4 \%)$ & 0,07 & $2(2,8 \%)$ & $5(3,9 \%)$ & 0,7 \\
CT $>240 \mathrm{mg} / \mathrm{dl}$ & $39(20,6 \%)$ & $30(9,3 \%)$ & 0,001 & $16(22,5 \%)$ & $20(15,6 \%)$ & 0,2 \\
cHDL $<40 \mathrm{mg} / \mathrm{dl}$ & $75(39,7 \%)$ & $97(30 \%)$ & 0,03 & $18(25,4 \%) \mathrm{f}$ & $19(14,8 \%)$ & 0,06 \\
TC $>150 \mathrm{mg} / \mathrm{dl}$ & $107(56,6 \%)$ & $129(40,9 \%)$ & 0,0001 & $36(50,7 \%)$ & $34(26,6 \%)$ & 0,001 \\
\hline
\end{tabular}

*Varón > 45 años o mujer > 55 años o menopausia; * * Infarto de miocardio o muerte súbita en familiar de primer grado varón antes de los 55 años de edad, o en familiar de primer grado mujer antes de los 65 años de edad; AF Cl: antecedentes familiares de cardiopatía isquémica prematura; ECV: enfermedad cardiovascular establecida; HTA: hipertensión arterial; CT: colesterol total; cHDL: colesterol de las lipoproteínas de alta densidad; TG: triglicéridos.

Diferencia significativa al comparar con varones con lipodistrofia, ${ }^{a} p: 0,03 ;{ }^{b} p: 0,04 ;{ }^{c} p: 0,003 ;{ }^{d} p: 0,0001 ;$ e p: 0,01; f p: 0,03.

$(12,13)$. En los varones predominó el patrón de lipoatrofia, mientras que en las mujeres fueron más prevalentes las formas con lipoacúmulo, al igual que han referido otros autores $(15,25)$. Cuando se analizaron los factores de riesgo cardiovascular según la presencia o no de lipodistrofia, se observó que los varones con distribución anómala de la grasa corporal mostraron respecto a los varones sin lipodistrofia, un perfil de alto riesgo cardiovascular consistente en una mayor prevalencia de hipertensión arterial, hipercolesterolemia, hipertrigliceridemia y bajas concentraciones de cHDL. Estos hallazgos son concordantes con descripciones previas y sugieren la existencia de un estado subyacente de resistencia a la insulina como mecanismo patogenético central $(14,17)$. Sin embargo, en el grupo de las mujeres, la lipodistrofia sólo se asoció a una mayor presencia de obesidad central e hipertrigliceridemia. Un reciente estudio corrobora estos hallazgos, y demuestra que el índice cintura/cadera, como parámetro antropométrico de distribución anómala de la grasa, se asocia de forma significativa con marcadores de inflamación como la proteína $\mathrm{C}$ reactiva, la interleucina 6 y la adiponectina en mujeres con infección por el VIH (23). El análisis comparativo de varones y mujeres con lipodistrofia, siguió revelando un contexto más aterogénico para los varones, por criterios de edad, presión arterial y concentración de cHDL. Sin embargo, la mujer con lipodistrofia presentó una considerable agregación de factores de riesgo cardiovascular, entre los que cabe destacar una mayor prevalencia de consumo de cigarrillos, antecedentes familiares de cardiopatía isquémica prematura y de obesidad central.

Las limitaciones del presente estudio se derivan sobre todo de su diseño transversal, por lo que no debemos olvidar la posible variabilidad temporal que pueden experimentar los parámetros evaluados y que los hallazgos descritos represen- tan sólo asociaciones y no implican causalidad. En relación a la evaluación de la lipodistrofia, la utilización de criterios clínicos podría infraestimar su prevalencia, ya que ésta es más frecuente en sus formas leves o moderadas (26). Sin embargo, no existe todavía consenso para la utilización de alguna de las técnicas de medida de la grasa corporal por la falta de estandarización (14), y aunque se dispone de una definición de lipodistrofia que incluye datos demográficos, clínicos, analíticos y de pruebas de imagen, ésta resulta compleja y de difícil aplicación en la práctica clínica (27).

En conclusión, el presente estudio demuestra que el impacto del sexo en el síndrome de lipodistrofia en los pacientes con infección por el VIH no sólo afecta al patrón de lipodistrofia, sino también al perfil de riesgo cardiovascular. En este sentido, aunque los hallazgos descritos pueden interpretarse como aparentemente ventajosos para la mujer, quisiéramos remarcar dos aspectos de gran trascendencia. En primer lugar, hemos utilizado como comparativo los varones con lipodistrofia e infección por el VIH, no población femenina aparentemente sana, y por otra parte no hay que olvidar la relación existente entre la obesidad central y el aumento de riesgo cardiovascular, tanto en la población general como en la población infectada por el VIH $(4,6,7,15)$. En base a la mayor esperanza de vida junto a la creciente exposición a la terapia antirretroviral es fácil pronosticar un incremento de su riesgo cardiovascular en las mujeres con infección por VIH, asociado a la edad y a la menopausia durante los próximos años. Desde un punto de vista terapéutico y dejando a parte las posibles sustituciones de fármacos antirretrovirales debemos recordar las evidencias disponibles a cerca de la importancia del cambio de estilo de vida, especialmente en situación de obesidad central para la población con y sin infección por VIH. 


\section{Bibliografía}

1. Tomás Abadal L. Riesgo cardiovascular en la menopausia: mito, paradoja o realidad. Importancia de las observaciones clínicas frente a la interpretación de los datos estadísticos. Rev Esp Cardiol 1999; 52: 463-6.

2. Polk DM, Naqvi TZ. Cardiovascular disease in women: sex differences in presentation, risk factors, and evaluation. Curr Cardiol Rep 2005; 7 : 166-172.

3. Marrugat J, Sala J, Masià R, Pavesi M, Gines G, Valle V, et al. Mortality differences between men and women following first myocardial infarction. JAMA 1998; 16: 1405-9.

4. Hochman JS, Tamis JE, Thompson TD, Weaver WD, White HD, Van de Werf F, et al. Sex, clinical presentation, and outcome in patients with acute coronary syndromes. Global Use of Strategies to Open Occluded Coronary Arteries in Acute Coronary Syndromes IIb Investigators. N Engl J Med 1999; 34: 226-32.

5. Rexrode KM, Carey VJ, Hennekens CH, Walters EE, Colditz GA, Stampfer MJ, et al. Abdominal adiposity and coronary heart disease in women. JAMA 1998; 280: 1843-8.

6. Rexrode KM, Buring JE, Manson JE. Abdominal and total adiposity and risk of coronary heart disease in men. Int $\mathrm{J}$ Obes Relat Metab Disord 2001; 25: 1047-56.

7. Lawlor DA, Ebrahim S, Whincup M, Sterne J, Papacosta O, Wannamethee $\mathrm{G}$, et al. Sex differences in body fat distribution and carotid intima media thickness: Cross sectional survey using data from the British regional heart study. J Epidemiol Community Health 2004: 58; 700-4.

8. Palella FJ, Delaney KM, Moorman AC, Loveless MO, Fuhrer J, Satten GA, et al. Declining morbidity and mortality among patients with advanced HIV infection. N Engl J Med 1998; 338: 853-60.

9. Sterne JA, Hernan MA, Ledergerber B, Tilling K, Weber R, Sendi P, et al; Swiss HIV Cohort Study. Long-term effectiveness of potent antiretroviral therapy in preventing AIDS and death: a prospective cohort study. Lancet 2005; 366: 378-84

10. Carr A, Samaras K, Burton S, Law M, Freund J, Chisholm DJ, et al. A syndrome of peripheral lipodystrophy, hyperlipidaemia and insulin resistance in patients receiving HIV protease inhibitors. AIDS 1998;12: 51-8.

11. Mary-Krause M, Cotte L, Simon A, Partisani M, Costagliola D; the Clinical Epidemiology Group from the French Hospital Database. Increased risk of myocardial infarction with duration of protease inhibitor therapy in HIV-infected men. AIDS 2003; 17: 2479-86.

12. Friis-Moller N, Weber R, Reiss P, Thiébaut R, Kirk O, d'Arminio Monforte A, et al. Cardiovascular disease risk factors in HIV patients -association with antiretroviral therapy. Results from the DAD study. AIDS 2003; 17: 1179-93.

13. Carr A. HIV lipodystrophy: Risk factors, pathogenesis, diagnosis and management. AIDS 2003; 17 (Supl.1): 141-8.

14. Grinspoon S, Carr A. Cardiovascular risk and body-fat abnormalities in
HIV-infected adults. N Engl J Med 2005; 352: 48-62.

15. Galli M, Veglia F, Angarano G, Santambrogio S, Meneghini E, Gritti F, et al. Gender differences in antirretroviral-drug related adipose tissue alterations: Women are at higher risk than men and develop particular lipodystrophy patterns. J Acquir Immune Defic Syndr 2003; 34: 58-61.

16. The Data Collection on Adverse Events of Ant-HIV Drugs (DAD) Study Group. Combination antiretroviral therapy and the risk of myocardial infarction. N Engl J Med 2003; 340: 1993-2003.

17. Hadigan C, Meigs J, Corcoran C, Rietschel P, Piecuch S, Basgoz N, et al. Metabolic abnormalities and cardiovascular disease risk factors in adults with human immunodeficiency virus infection and lipodystrophy. Clin Infect Dis 2001; 32: 130-9.

18. National Cholesterol Education Program. Third Report of the National Cholesterol Education Program on Detection, Evaluation, and Treatment of High Blood Cholesterol in Adults (Adult Treatment Panel III). JAMA 2001; 285: 2486-97.

19. Chobanian AV, Bakris GL, Black HR, Cushman WC, Green LA, Izzo JL, et al; National High Blood Pressure Education Program Coordinating Committee. The Seventh Report of the Joint National Committee on Prevention, Detection, Evaluation, and Treatment of High Blood Pressure: the JNC 7 report. JAMA 2003; 289: 2560-72.

20. Anonymous. Report of the Expert Committee on the Diagnosis and Classification of Diabetes Mellitus. Diabetes Care 2003; 26 (Supl.1): S5-20.

21. Centers for Disease Control and Prevention: 1993 Revised classification system for HIV infection and expanded surveillance case definition for AIDS among adolescents and adults. MMWR 1992; 41 (RR-17): 1-19.

22. Tien PC, Cole SR, Williams CM, Li R, Justman JE, Cohen MH, et al. Incidence of lipoatrophy and lipohypertrophy in the women's integrance HIV study. J Acquir Immnune Defic Syndr 2003; 34: 461-6.

23. Dolan SE, Hadigan C, Killilea KM, Sullivan MP, Hemphill L, Lees RS, et al. Increased cardiovascular disease risk indices in HIV-Infected women. J Acquir Immune Defic Syndr. 2005;39: 44-54.

24. Howard AA, Floris-Moore M, Arnsten JH, Santoro N, Fleischer N, Lo $\mathrm{Y}$, et al. Disorders of glucose metabolism among HIV-infected women. Clin Infect Dis 2005; 40: 1492-9.

25. Jacobson D, Knox T, Spiegelman D, Skinner S, Gorbach S, Wanke C. Prevalence of, evolution of, and risk factors for fat atrophy and fat deposition in a cohort of HIV-infected men and women. Clin Infect Dis 2005; 40: 1837-45.

26. Miller J, Carr A, Emery S, Law M, Mallal S, Baker D, et al. HIV lipodystrophy: prevalence, severity and correlates of risk in Australia. HIV Medicine 2003; 4: 293-301.

27. Carr A, Emery S, Law M, Puls R, Lundgren JD, Powderly WG. An objective case definition of lipodystrophy in HIV-infected adults: A case-control study. Lancet 2003; 361: 726-35. 